\title{
Geometric approach to asymptotic expansion of Feynman integrals
}

\author{
A. Pak ${ }^{1}$, A. Smirnov ${ }^{1,2, a}$ \\ ${ }^{1}$ Institut für Theoretische Teilchenphysik, Karlsruhe Institute of Technology (KIT), 76128 Karlsruhe, Germany \\ ${ }^{2}$ Scientific Research Computing Center, Moscow State University, 119991 Moscow, Russia
}

Received: 4 March 2011 / Revised: 27 April 2011 / Published online: 12 April 2011

(C) The Author(s) 2011. This article is published with open access at Springerlink.com

\begin{abstract}
We present an algorithm that reveals relevant contributions in non-threshold-type asymptotic expansion of Feynman integrals about a small parameter. It is shown that the problem reduces to finding a convex hull of a set of points in a multidimensional vector space.
\end{abstract}

\section{Introduction}

Evaluation of Feynman integrals depending on multiple parameters is a notoriously difficult task. When direct computation fails, one resorts to studying asymptotics in various limits. In practice, a few first terms in the expansion may already suffice to reach the desired precision. However, expansion of a multi-loop integral may become non-trivial due to an interplay of parameters with the integration variables (components of loop momenta). Classification of relevant sectors in the integration space is itself a challenging problem $[1,2]$.

One important case is the asymptotic expansion in momenta and masses in the limits typical for the Euclidean space. This problem has been completely solved in terms of sums over subgraphs (for reviews, see Refs. [3, 4]). At least one automated tool $[5,6]$ implements this approach in practice. For a more general situation, including the limits appearing in the Minkowski space, there exists the universal strategy of expansion by regions in the momentum space $[1,2,7]$. In all known cases, it produces correct results, but a rigorous proof is still lacking. Typically, one manually analyzes a multi-scale problem, starting from simpler examples that can be checked against known analytical results or numerical estimates, computed e.g. with FIESTA [8] (later versions [9] of FIESTA may also evaluate a few first terms in a given asymptotic expansion.)

\footnotetext{
a e-mail: asmirnov57@yandex.ru
}

An important type of non-Euclidean expansions, the socalled threshold expansion [1], requires the most careful treatment. Relative scaling of terms becomes evident only in a specially chosen reference frame or with a certain routing of loop momenta. In what follows we suggest a very general approach to non-threshold asymptotic expansion, based on alpha-representation of integrals, and describe a simple practical algorithm.

\section{Expansion by regions and alpha-representation}

A thorough introduction to the expansion by regions and alpha-representation can be found elsewhere [7, 10]. Here we briefly introduce the basic notation with a trivial example.

Consider a family of one-loop propagator-type integrals in the Euclidean space:

$$
\begin{gathered}
I_{1}\left(a_{1}, a_{2} ; p^{2}, m^{2}\right)=\int \frac{d^{D} k}{(2 \pi)^{D}} \frac{1}{D_{1}^{a_{1}} D_{2}^{a_{2}}}, \\
D_{1}=k^{2}+m^{2}, D_{2}=(k+p)^{2}+m^{2} .
\end{gathered}
$$

A specific integral is determined by the (integer) exponents $a_{1}$ and $a_{2}$ and depends on the two parameters, $m^{2}$ and $p^{2}$. The structure of the expansion does not depend on $a_{1}$ and $a_{2}$ and we will not focus on those exponents in the following discussion.

Let us consider the asymptotics of $I_{1}\left(a_{1}, a_{2} ; p^{2}, m^{2}\right)$ in the limit when $\left|p^{2}\right| \gg m^{2}$, or $\rho=\left|m^{2} / p^{2}\right| \ll 1$. The naive Taylor expansion does not capture the complete asymptotic behaviour since the integration variables (components of $k$ ) span all values from $-\infty$ to $+\infty$, and in particular may be as small as $m$ or as large as $\sqrt{\left|p^{2}\right|}$.

The prescription in this case is to find regions, or scalings of momentum components that after the expansion provide 
non-zero contributions. In each region, we first Taylor expand the integrand and drop the scaling restrictions. In our example, there are three non-zero regions, summarised in Table 1. For example, in the region (c) one expands the factor $D_{2}^{-a_{2}}$ as follows:

$$
\begin{aligned}
& I_{1}\left(a_{1}, a_{2} ; p^{2}, m^{2}\right)=\int_{k_{\mu} \sim m} \frac{d^{D} k}{(2 \pi)^{D}} \frac{1}{\left(k^{2}+m^{2}\right)^{a_{1}}}, \\
& {\left[\frac{1}{\left(p^{2}\right)^{a_{2}}}-\frac{a_{2}\left(k^{2}+2 k p+m^{2}\right)}{\left(p^{2}\right)^{a_{2}+1}}+\cdots\right]+\text { other regions }} \\
& \quad=\int \frac{d^{D} k}{(2 \pi)^{D}} \frac{1}{\left(k^{2}+m^{2}\right)^{a_{1}}\left(p^{2}\right)^{a_{2}}} \\
& \quad+\int \frac{d^{D} k}{(2 \pi)^{D}} \frac{(\ldots)}{\left(k^{2}+m^{2}\right)^{a_{1}}\left(p^{2}\right)^{a_{2}+1}}+\cdots \\
& \quad+\text { other regions. }
\end{aligned}
$$

In the last line, we dropped the restriction on $k$ and collected the contributions proportional to the same power of $\rho$ together. Every coefficient then is represented by a sum of single-scale Feynman integrals with simpler denominator factors, various denominator exponents and some numerators. The non-trivial statement is that the double-counting which could have been introduced disappears in the sum of all regions. For the purpose of the following discussion we assume that the tensor reduction of numerators and the proliferation of terms can be managed; we will focus on the

Table 1 Regions (b-d) of expansion of a double-scale integral (a) in the momentum space and in the alpha-representation

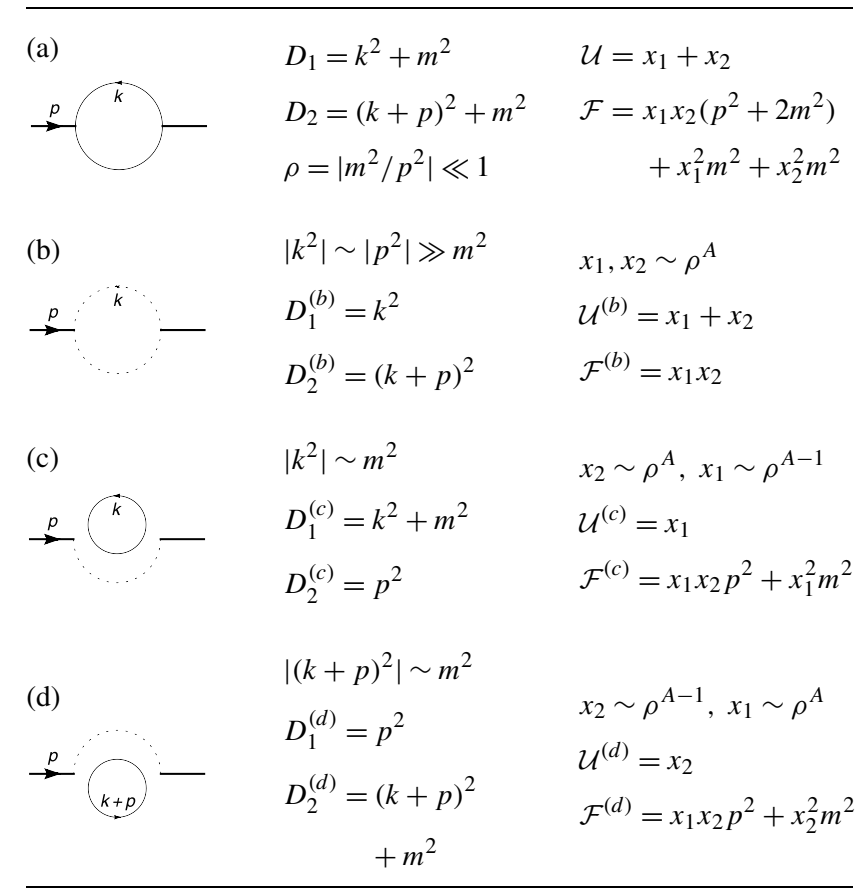

transformations of denominator factors in every region (e.g. $(k+p)^{2}+m^{2} \rightarrow p^{2}$ in the example above).

Some types of asymptotic expansion may require more elaborate choice of regions. For example, $k_{0}$ may scale differently from $k_{i}$ or some combination of components may have a separate scale (this happens, e.g., in Sudakov limits). It is thus desirable to have an explicitly covariant formalism to identify regions, independent of the frame choice and the routing of momenta. For that purpose, we may switch to the alpha-representation of integrals. We re-write an integral with $n$ lines (denominator factors) over $D$-dimensional loop momenta as an integral over $n$ positive parameters $x_{1}, \ldots, x_{n}$. Information about the graph is then encoded in the two homogeneous polynomials, $\mathcal{U}$ and $\mathcal{F}$. For example, the integral of (1) is

$$
\begin{aligned}
I_{1}\left(a_{1}, a_{2} ; p^{2}, m^{2}\right) \\
=\frac{\Gamma\left(a_{1}+a_{2}-D / 2\right)}{\Gamma\left(a_{1}\right) \Gamma\left(a_{2}\right)} \\
\quad \times \int_{0}^{\infty} d x_{1} d x_{2} \delta\left(1-x_{1}-x_{2}\right) x_{1}^{a_{1}-1} x_{2}^{a_{2}-1} \\
\quad \times \mathcal{U}^{a_{1}+a_{2}-D} \mathcal{F}^{D / 2-a_{1}-a_{2}}, \\
\mathcal{U}=x_{1}+x_{2}, \\
\mathcal{F}=x_{1} x_{2}\left(p^{2}+2 m^{2}\right)+x_{1}^{2} m^{2}+x_{2}^{2} m^{2} .
\end{aligned}
$$

The dependence on $a_{1}$ and $a_{2}$ and the delta-function do not play any role in the following discussion (alpharepresentation may be formulated without this delta function). It is, however, important that this integral is regulated by the non-integer dimension $D$. In what follows, we implicitly require that all considered integrals exist for some choice of $D$.

The strategy of expansion in kinematic regions may also be formulated in the alpha-representation $[2,7]$. Instead of finding the scaling behaviour of loop momentum components, we determine the relative scaling of every parameter $x_{i}$ in terms of the small parameter, and that directly corresponds to the scale of the $i$-th line (denominator factor) of the original integral. The expansion removes non-leading terms from $\mathcal{U}$ and $\mathcal{F}$, and the resulting polynomials describe the integrals obtained by the expansion in the momentum space. The last column of Table 1 demonstrates the scaling of alpha-parameters and the polynomials in each expansion region.

Using the language of alpha-parameters, we may attempt to define the difference between the threshold and non-threshold asymptotic expansion. As an example, let us again consider the integral of (1) in the threshold limit $y=m^{2}+\frac{p^{2}}{4} \ll m^{2}$ (that naturally implies that $p^{2}<0$, i.e. this limit is essentially non-Euclidean). Choosing the 
frame where $p=\left(p_{0}, \boldsymbol{0}\right)$ and re-routing the loop momentum $k=\left(k_{0}, \mathbf{k}\right)$, we re-write the denominator factors as $D_{1}=k_{0}^{2}+\mathbf{k}^{2}+k_{0} p_{0}+y$ and $D_{2}=k_{0}^{2}+\mathbf{k}^{2}-k_{0} p_{0}+y$.

It is known that this integral has two non-vanishing regions. The first "hard" region is characterised by $k \sim m$. The second ("potential") region corresponds to the scaling $k_{0} \sim y / m,|\mathbf{k}| \sim \sqrt{y}$. In terms of alpha-parameters, we should be able to determine those regions by inspecting the polynomial $\mathcal{F}=y\left(x_{1}+x_{2}\right)^{2}-\frac{p^{2}}{4}\left(x_{1}-x_{2}\right)^{2}$. In the "hard" region, only the second term survives due to the large factor $p^{2}$, which is similar to the previous "non-threshold" example. To identify the "potential" region, we must notice that there exists a thin layer in the integration space near the surface $x_{1}=x_{2}$, where the second term has the same scaling as the first, and this contribution does not vanish.

In a similar way, more complex threshold expansions contain contributions which depend on cancellations between the terms in the expanded $\mathcal{F}$ along some non-trivial surface and not at zero or infinity. The exact shape of this surface depends on numerical coefficients in $\mathcal{F}$, and in some cases also on the relations between kinematic invariants. Presently we do not know a general rule to identify such surfaces and find substitutions revealing the corresponding regions. In this paper we focus on the "usual" regions that can be determined by examining independently the monomials in $\mathcal{U}$ and $\mathcal{F}$. However limited, this problem is still important for many applications. We have observed that this strategy reliably detects the typical regions appearing in the expansion in small or large masses or large momenta, including Sudakov-type contributions that correspond to collinear, soft, and ultra-soft regions.

\section{General formalism}

We consider an $l$-loop Feynman integral

$I\left(a_{1}, \ldots, a_{n}\right)=\int \frac{d^{D} k_{1} \cdots d^{D} k_{l}}{(2 \pi)^{l D} D_{1}^{a_{1}} \cdots D_{n}^{a_{n}}}$,

which depends on $n$ exponents $a_{1}, \ldots, a_{n}$, scalar products of $e$ external momenta $p_{1}, \ldots, p_{e}$ and parameters (such as masses) in denominator factors $D_{i}$. The latter must be quadratic in momenta but other than that may have any form, e.g. correspond to a massive, such as $-\left(k_{i}+p_{j}\right)^{2}+m_{k}^{2}-i 0$, or a static propagator, such as $\left(-2 k_{i} p_{j} \pm i 0\right)$. The alpharepresentation for this integral has a general structure

$$
\begin{aligned}
I\left(a_{1}, \ldots, a_{n}\right)= & c \int_{0}^{1} d x_{1} \cdots d x_{n} \delta\left(1-x_{1}-\cdots-x_{n}\right) \\
& \times x_{1}^{a_{1}-1} \cdots x_{n}^{a_{n}-1} \mathcal{U}^{a} \mathcal{F}^{b},
\end{aligned}
$$

where coefficient $c$ and exponents $a$ and $b$ depend only on $l, D$, and $a_{i} . \mathcal{U}$ and $\mathcal{F}$ are homogeneous polynomials (of order $l$ and $l+1$, respectively) of integration variables $x_{i}$, and
$\mathcal{F}$ also depends on the kinematic invariants. If the denominators $D_{i}$ correspond to some graph and have a standard form $-k^{2}+m^{2}-i 0$ (in Minkowski space), then the functions $\mathcal{U}$ and $\mathcal{F}$ can be read off the graph in terms of trees and 2-trees [10]. In a more general case, one may obtain $\mathcal{U}$ and $\mathcal{F}$ with the program UF [11]. In what follows, we will only discuss the properties of $\mathcal{U}$ and $\mathcal{F}$ that are independent of the specific indices $a_{i}$.

In dimensional regularisation, "scaleless" integrals (having no inherent scale) turn to zero. More specifically, an integral is scaleless if it is possible to re-scale some loop momenta or their components so that the result remains proportional to the original integral, or $D_{i}\left(\left\{k_{j}\right\},\left\{a k_{i}\right\}\right)=$ $a^{u_{i}} D_{i}(\{k\})$, with some subset $\left\{k_{i}\right\}$ of integration momenta. In particular, massless vacuum bubbles vanish:

$I=\int \frac{d^{D} k}{\left(k^{2}\right)^{n}}=\int \frac{d^{D}(\alpha k)}{\left((\alpha k)^{2}\right)^{n}}=\alpha^{D-2 n} I=0$.

In terms of the alpha-representation (5), a similar statement applies to homogeneity of $\mathcal{U}$ and $\mathcal{F}$ with respect to a subset $\{B\}$ of integration variables $x_{i}(\{B\}$ should not coincide with the full set of $\left.\left\{x_{i}\right\}\right)$. Integrals vanish if $\mathcal{U}\left(\left\{x_{j}\right\},\left\{a x_{i}\right\}\right)=a^{u} \mathcal{U}(\{x\})$ and $\mathcal{F}\left(\left\{x_{j}\right\},\left\{a x_{i}\right\}\right)=a^{f} \mathcal{F}(\{x\})$, $i \in\{B\}$, with some scaling dimensions $u$ and $f$.

In order to avoid separate treatment of $\mathcal{U}$ and $\mathcal{F}$, one may consider the product $\mathcal{U F}$ that incorporates the scaling and asymptotic properties of both factors (but may contain many terms).

\section{Geometric interpretation of asymptotic expansion}

Let us start with some integral in the alpha-representation (5) with integration variables $x_{1}, \ldots, x_{n}$ and a small expansion parameter $\rho$. Each of $M$ terms in $\mathcal{F}$ corresponds to a vector of $n+1$ exponents (we here neglect common factors and numeric coefficients, irrelevant to the non-threshold expansion):

$\rho^{r_{0}} x_{1}^{r_{1}} \cdots x_{n}^{r_{n}} \rightarrow\left(r_{0}, r_{1}, \ldots, r_{n}\right)$,

and $\mathcal{F}$ corresponds to a set $\{F\}$ of $M$ points in $(n+1)$ dimensional vector space. Due to homogeneity of $\mathcal{F}$, all these points belong to an $n$-dimensional hyperplane $r_{1}+$ $\cdots+r_{n}=l+1$, parallel to the 0 -th axis (the axis of $r_{0}$ ).

Terms of $\mathcal{U}$ have no explicit powers of $\rho$ in the coefficients. The corresponding set $\{U\}$ is thus confined to an $(n-2)$-dimensional hyperplane $r_{0}=0, r_{1}+\cdots+r_{n}=l$. If we fix the scales of alpha-parameters as $x_{i} \sim \rho^{v_{i}}$, then the scale of a monomial can be found as $\rho^{r_{0}} x_{1}^{r_{1}} \cdots x_{n}^{r_{n}} \sim$

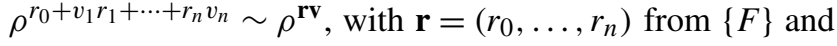
$\mathbf{v}=\left(1, v_{1}, \ldots, v_{n}\right)$. Graphically, $\mathbf{r v}$ represents the length of a projection that vector $\mathbf{r}$ has on the direction $\mathbf{v}$. 
Generally, any choice of $\mathbf{v}$ corresponds to some hierarchy between $x_{i}$, but most of such choices lead to zero (scaleless) integrals. The few directions that lead to scaleful integrals determine the regions of expansion that we seek. The terms in $\mathcal{F}$ that remain after the expansion are all characterised by the same scale in powers of $\rho$. In terms of the corresponding subset of points $\left\{F^{\prime}\right\}$, they feature the same value of the projection on $\mathbf{v}$. In other words, all these points lie in the same hyperplane orthogonal to $\mathbf{v}$.

The points corresponding to the neglected terms will be located "above" this hyperplane (since $\mathbf{v}$ always points "up" with respect to the 0 -th axis). If we now introduce an envelope, or "convex hull" of the set $\{F\}$, then points $\left\{F^{\prime}\right\}$ must belong to one of its facets. The corresponding $\mathbf{v}$ then is the normal vector to that facet. (Technically, these points may also belong to a lower-dimensional "ridge" between the facets, but as will be shown further, such expansions give vanishing contributions.)

To summarise, we re-stated the problem of finding the regions as the problem of finding the "bottom" facets of the convex hull, built for the vector set $\{F\}$ (or $\{U F\}$, if we want to avoid separate analysis of $\{U\})$.

\subsection{Trivial example}

In Fig. 1 we present the vectors corresponding to the example in (1). The three terms of $\{F\}$ are denoted with crossed points and the two terms of $\{U\}$-with diamonds.

Relating the three expansion regions of Table 1 to graphics in Fig. 1, we find the corresponding points and vectors (points as denoted in the figure):
$\mathrm{b}: \mathbf{v}=(1,0,0)$
$\left\{F^{\prime}\right\}=(C)$,
$\left\{U^{\prime}\right\}=(D, E)$,
c: $\mathbf{v}=(1,1,-1)$,
$\left\{F^{\prime}\right\}=(A, C)$,
$\left\{U^{\prime}\right\}=(D)$,
$\mathrm{d}: \mathbf{v}=(1,-1,1)$,
$\left\{F^{\prime}\right\}=(B, C)$,
$\left\{U^{\prime}\right\}=(E)$.

Here we exploit the freedom to re-scale all $x_{i}$ by the same power of $\rho$, i.e. shift $\mathbf{v}$ by any vector $\mathbf{a}=(0, A, \ldots, A)$. If $\mathbf{v}$ corresponds to a region, then $\mathbf{v}^{\prime}=\mathbf{v}+\mathbf{a}$ determines the same region. For example, $\mathbf{v}^{\prime}=(1,2,0)$ also corresponds to the region (c) above. It is generally convenient to choose

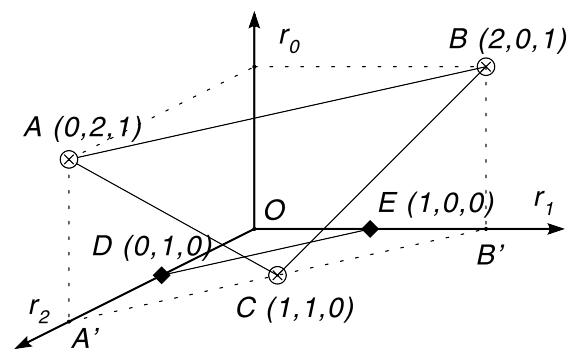

Fig. 1 Graphical representation of sets $\{F\}$ (crossed points) and $\{U\}$ (diamonds) corresponding to the integral of (1) $\mathbf{v}$ parallel to the plane where points $\{F\}$ are confined, i.e. orthogonal to the vector $(0,1, \ldots, 1)$.

\subsection{Scaleless regions}

Normally, only a few scaling choices produce non-zero regions. In our example, the choice $x_{1} \sim \rho^{2}, x_{2} \sim \rho^{0}$, leading to $\mathcal{U}=x_{2}, \mathcal{F}=x_{2}^{2} m^{2}$, or $\left\{F^{\prime}\right\}=(A),\left\{U^{\prime}\right\}=(D)$, corresponds to a scaleless integral. As discussed above, this implies an existence of a scaling leaving both $\mathcal{U}$ and $\mathcal{F}$ invariant up to a pre-factor (in this case, $x_{1} \rightarrow a x_{1}$ ).

The requirement that a region does not vanish can be easily formulated in the geometrical language. Consider the polynomial $\mathcal{U F}$ and the corresponding set of points $\{U F\}$. After the expansion with the chosen scalings, we are left with its subset $\left\{U^{\prime} F^{\prime}\right\}$. Numeric coefficients and kinematic invariants are irrelevant to the scalefulness of a region, and we get rid of them by projecting $\left\{U^{\prime} F^{\prime}\right\}$ on the plane $r_{0}=0$. The thus obtained set of points $\left\{U^{\prime} F_{0}^{\prime}\right\}$ belongs to the $(n-1)$-dimensional hyperplane.

Scalelessness implies that all terms of the polynomial $\mathcal{U}^{\prime} \mathcal{F}^{\prime}$ are homogeneous with respect to a certain re-scaling, $x_{i} \rightarrow x_{i} \rho^{w_{i}}$. This implies that all points of $\left\{U^{\prime} F_{0}^{\prime}\right\}$ must belong to an orthogonal space of the corresponding rescaling vector $\mathbf{w}_{h}=\left(0, w_{1}, \ldots, w_{n}\right)$, which is not parallel to the trivial re-scaling direction $(0,1, \ldots, 1)$. In other words, $\left\{U^{\prime} F_{0}^{\prime}\right\}$ may exist in at most $(n-2)$-dimensional subspace, and its $(n-1)$-dimensional volume is zero. The latter property can be very useful to determine vanishing integrals. However, it is also easy to see that the "bottom" facets of the convex hull for $\{U F\}$ automatically correspond to scaleful regions: their dimension is $(n-1)$ by construction (otherwise they are considered "ridges" or "vertices"), and they (being "bottom") are not orthogonal to the plane $r_{0}=0$, thus the projection has non-zero volume.

\subsection{General procedure}

Finally, we can formulate the way to determine the expansion regions. We start by building the set of points $\{U F\}$. Next, we find the $n$-dimensional convex hull $\mathcal{C}$ of the set $\{U F\}$ in the $n$-dimensional plane $r_{1}+\cdots+r_{n}=l+1$, using any preferred algorithm. The implementation that we chose, QHull [12], does not allow building hulls of dimensionality lower than the dimension of vector space. Thus, one has to introduce local coordinate system and deal with non-integer coordinate values. However, it is also possible to project $\{U F\}$ along any of axes $r_{i}, i \neq 0$, e.g. consider $(n-1)$-dimensional points $\mathbf{r}_{\|}=\left(r_{0}, r_{1}, \ldots, r_{n-1}\right)$. Convex hull $\mathcal{C}^{\prime}$ built for this projection will be the projection of the "true" convex hull $\mathcal{C}$. Its dimensions will be stretched but the correspondence of the points to the facets and the vertices will persist. 
From the $(n-1)$-dimensional facets of $\mathcal{C}$ we then select the "bottom", i.e. facets with normal vectors $\mathbf{v}$ pointing "up", with $v_{0}>0$. For each of those "bottom" facets, we choose the normal vector $\mathbf{v}$ such that $v_{0}=1$. Its components 1 to $n$ represent the relative scales of alpha-parameters $x_{i}$ and thus uniquely determine an expansion region.

\section{Less trivial example}

Let us consider the integral in Fig. 2, this time defined in the Minkowski space (this example was first considered in [2] and [7], Chap. 10):

$$
\begin{aligned}
& I_{2}\left(a_{1}, \ldots, a_{6} ; s, m^{2}, M^{2}\right)=\int \frac{d^{D} k_{1} d^{D} k_{2}}{(2 \pi)^{2 D} D_{1}^{a_{1}} \cdots D_{6}^{a_{6}}} \\
& D_{1}=\left(p_{1}-k_{1}-k_{2}\right)^{2}-M^{2} \\
& D_{2}=\left(p_{1}-k_{2}\right)^{2}-M^{2} \\
& D_{3}=\left(p_{2}+k_{1}+k_{2}\right)^{2}-m^{2}, \\
& D_{4}=\left(p_{2}+k_{2}\right)^{2}-m^{2} \\
& D_{5}=k_{1}^{2}, \quad D_{6}=k_{2}^{2} \\
& p_{1}^{2}=M^{2}, \quad p_{2}^{2}=m^{2}, \\
& \left(p_{1}+p_{2}\right)^{2}=s, \quad s, M^{2} \gg m^{2} .
\end{aligned}
$$

With $S=m^{2}+M^{2}-s=-2 p_{1} p_{2}$, its alpha-representation reads:

$$
\begin{aligned}
\mathcal{U}= & x_{1} x_{2}+x_{3} x_{2}+x_{5} x_{2}+x_{1} x_{4}+x_{3} x_{4} \\
& +x_{1} x_{5}+x_{3} x_{5}+x_{4} x_{5}+x_{1} x_{6}+x_{3} x_{6}+x_{5} x_{6}, \\
\mathcal{F}= & M^{2} x_{1}^{2} x_{2}+M^{2} x_{1}^{2} x_{4}+M^{2} x_{1}^{2} x_{5}+M^{2} x_{1} x_{2}^{2} \\
& +M^{2} x_{2}^{2} x_{3}+M^{2} x_{2}^{2} x_{5}+M^{2} x_{1}^{2} x_{6}+2 M^{2} x_{1} x_{2} x_{5} \\
& +m^{2} x_{2} x_{3}^{2}+m^{2} x_{3}^{2} x_{4}+m^{2} x_{1} x_{4}^{2}+m^{2} x_{3} x_{4}^{2} \\
& +m^{2} x_{3}^{2} x_{5}+m^{2} x_{4}^{2} x_{5}+m^{2} x_{3}^{2} x_{6}+2 m^{2} x_{3} x_{4} x_{5} \\
& +S x_{1} x_{2} x_{3}+S x_{1} x_{2} x_{4}+S x_{1} x_{3} x_{4}+S x_{1} x_{3} x_{5} \\
& +S x_{1} x_{4} x_{5}+S x_{1} x_{3} x_{6}+S x_{2} x_{3} x_{4}+S x_{2} x_{3} x_{5} \\
& +S x_{2} x_{4} x_{5} .
\end{aligned}
$$

For simplicity, let us analyze $\mathcal{F}$ instead of the product $\mathcal{U} \mathcal{F}$. Choosing $m$ as the small parameter and preserving the order of terms, in the 7-dimensional space we have 25

Fig. 2 Double-scale two-loop vertex integral

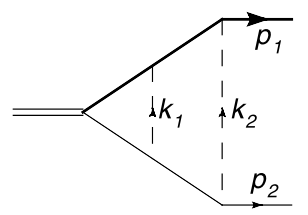

points: $\{F\}=(0,2,1,0,0,0,0),(0,2,0,0,1,0,0),(0,2,0$, $0,0,1,0), \ldots$.

To build the 6-dimensional convex hull, we project $\{F\}$ along the 6th axis: $\{F\}_{p}=(0,2,1,0,0,0),(0,2,0,0,1,0)$, ... The hull of $\{F\}_{p}$ has 18 facets, four of which belong to the "bottom". Restoring the 7-dimensional normal vectors with unit 0 th components, we find: $\mathbf{v}_{1}=$ $(1,0,-2,-2,-2,-2,-4), \mathbf{v}_{2}=(1,0,0,-2,-2,-2,-2)$, $\mathbf{v}_{3}=(1,0,0,-2,0,0,0), \mathbf{v}_{4}=(1,0,0,0,-2,0,-2)$. Since $\mathcal{U}$ is scaleful, we also have to add the "hard" region: $\mathbf{v}_{0}=$ $(1,0,0,0,0,0,0)$. (The latter would appear automatically, had we considered the product $\mathcal{U} \mathcal{F}$.)

For illustration, let us consider the most non-trivial "ultrasoft-collinear" region corresponding to $\mathbf{v}_{1}$, or the scaling of alpha-parameters $x_{1} \sim m^{0}, x_{2} \sim 1 / m^{2}, x_{3} \sim 1 / m^{2}$, $x_{4} \sim 1 / m^{2}, x_{5} \sim 1 / m^{2}$, and $x_{6} \sim 1 / m^{4}$. First, we may check that $\mathbf{v}_{1}$ is indeed orthogonal to the plane containing the points $5,6,15,22,23,24$, and 25 from $\{F\}$ (in the order as in (9)). Those points correspond to the terms remaining in $\mathcal{F}^{\prime}$ after the expansion: $M^{2} x_{2}^{2} x_{3}+M^{2} x_{2}^{2} x_{5}+\cdots$.

In the momentum space, the interpretation becomes clear only in the special reference frame, where $p_{1}=(M, \mathbf{0}$, $0), p_{2}=M n_{+}+\left(\frac{m^{2}}{M}\right) n_{-}$, and $n_{ \pm}=(1 / 2, \mathbf{0}, \mp 1 / 2)$. We also decompose the first loop momentum in plus- and minus-parts, $k_{1}=\left(k_{+}+k_{-}, \mathbf{k}, k_{+}-k_{-}\right)$. To reproduce the "ultrasoft-collinear" region, we should prescribe the following scales to the components of loop momenta: $k_{+} \sim m^{2} / M$, $k_{-} \sim M, \mathbf{k} \sim m, k_{2} \sim m^{2} / M$.

After the expansion, the denominator factors scale as: $D_{1} \sim M^{2}, D_{2}, D_{3}, D_{4}, D_{5} \sim m^{2}, D^{6} \sim m^{4} / M^{2}$. One can easily see how the powers of $m$ here correspond to the components of $\mathbf{v}_{1}$.

\section{Implementation}

We wrote a Mathematica program that determines the expansion regions of a given Feynman integral based on the procedure described above. The general problem of building a convex hull of $M$ points in $n$ dimensions is wellknown in computational geometry; we employ the algorithm quickhul1 [12] that has complexity $\mathcal{O}\left(M^{\lfloor d / 2\rfloor}\right)$. It is sufficient when the number of lines is not too large; for example, finding 11 expansion regions of a 4-loop integral with 10 lines takes about 10 seconds on a laptop PC.

The program has been checked against some non-trivial examples discussed in [7] and [13]. The code can be downloaded from [11].

In order to run the program, one has to install the opensource package QHull [12]. If the executable is not in the current directory, Options [QHull] must be updated in the file asy.m. The program is loaded with command $<<$ asy.m. 
The main function is AlphaRepExpand $[k s, d s, c s$, $\mathrm{hi}$ ], where ks is the list of loop momenta (e.g., $\{\mathrm{v} 1, \mathrm{v} 2\})$, $\mathrm{ds}$ are the denominators (e.g., $\left\{(\mathrm{p} 1-\mathrm{v} 1-\mathrm{v} 2)^{\wedge} 2-\mathrm{M}^{\wedge} 2\right.$, $(\mathrm{p} 1-\mathrm{v} 2)^{\wedge} 2-\mathrm{M}^{\wedge} 2,(\mathrm{p} 2+\mathrm{v} 1+\mathrm{v} 2)^{\wedge} 2-\mathrm{m}^{\wedge} 2,(\mathrm{p} 2+\mathrm{v} 2)^{\wedge} 2$ $\left.\left.-m^{\wedge} 2, v 1^{\wedge} 2, v 2^{\wedge} 2\right\}\right)$, cS contains the kinematic constraints (e.g., $\quad\left\{\mathrm{p} 1^{\wedge} 2->\mathrm{M}^{\wedge} 2, \mathrm{p} 22^{\wedge} 2->\mathrm{m}^{\wedge} 2, \mathrm{p} 1 * \mathrm{p} 2->-\right.$ $S / 2\}$ ), and $h i$ represents the scalings of kinematic invariants with respect to the small parameter $x$ (e.g., $\left.\left\{\mathrm{M}->\mathrm{x}^{\wedge} 0, \mathrm{~S}->\mathrm{x}^{\wedge} 0, \mathrm{~m}->\mathrm{x}^{\wedge} 1\right\}\right)$.

The output is a list of vectors specifying the scales of the alpha-parameters factors, or the non-zero components of vectors $\mathbf{v}_{i}$. For the example of Sect. 5, the output is $\{\{0,-2,-2,-2,-2,-4\},\{0,0,-2,-2,-2,-2\}\}$, $\{\{0,0,-2,0,0,0\},\{0,0,0,-2,0,2\},\{0,0,0\}$, $\{0,0,0\}\}$, corresponding to the regions (us-1c), (1c-h), (h-1c), (1c-1c), and (h-h). These regions can be understood by analogy to the example of Sect. 5 .

\section{Conclusion}

We present an algorithm to find the relevant regions of expansion for a Feynman integral in a given limit of momenta and masses.

The algorithm is implemented in Wolfram Mathematica language and uses open-source package QHul1. The program and examples can be downloaded from our web- page. In the future, we plan to extend the code in order to apply it to more general parametric integrals.

Acknowledgement We would like to thank V.A. Smirnov for suggesting the topic and constant help, and M. Steinhauser for the very useful comments.

Open Access This article is distributed under the terms of the Creative Commons Attribution Noncommercial License which permits any noncommercial use, distribution, and reproduction in any medium, provided the original author(s) and source are credited.

\section{References}

1. M. Beneke, V.A. Smirnov, Nucl. Phys. B 522, 321 (1998)

2. V.A. Smirnov, Phys. Lett. B 465, 226 (1999)

3. F. Tkachov, Sov. J. Part. Nucl. 25, 649 (1994)

4. V.A. Smirnov, Mod. Phys. Lett. A 10, 1485 (1995)

5. T. Seidensticker, hep-ph/9905298 (1999)

6. R. Harlander, T. Seidensticker, M. Steinhauser, Phys. Lett. B 426, 125 (1998)

7. V.A. Smirnov, Springer Tracts Mod. Phys. 177, 1 (2002)

8. A.V. Smirnov, M.N. Tentyukov, Comput. Phys. Commun. 180, 735 (2009)

9. A.V. Smirnov, V.A. Smirnov, M. Tentyukov, Comput. Phys. Commun. 182, 790 (2011)

10. N.N. Bogolyubov, D.V. Shirkov, Intersci. Monogr. Phys. Astron, vol. 3 (Interscience, New York, 1959), pp. 1-720

11. http://www-ttp.particle.uni-karlsruhe.de/ asmirnov/Tools-UF.htm

12. http://www.qhull.org

13. A. Pak, A. Czarnecki, Phys. Rev. D 78, 114015 (2008) 